\title{
Debye Frequency-extended Waveguide Permittivity Extraction for High Complex Permittivity Materials. Concrete Setting Process Characterization
}

\author{
G. González-López, S. Blanch, J. Romeu, Fellow, IEEE, and L. Jofre, Fellow, IEEE
}

\begin{abstract}
A waveguide with central frequency $868 \mathrm{MHz}$ is used in transmission/reflection operation regime to accurately measure the behaviour of the complex permittivity of high complex permittivity granular materials and it has been frequencyextended up to $3 \mathrm{GHz}$ using the Debye fitted relaxation model. It is shown that for highly granular high permittivity materials a waveguide based transmission/reflection technique is necessary to reduce the uncertainty of the extracted permittivity values. The technique is first described and validated with isopropyl alcohol and then applied to the characterization of cement based materials. This paper provides accurate data on the evolution of the complex permittivity of concrete and mortar from the moment of pouring until air dried condition is achieved.
\end{abstract}

Index Terms-relative permittivity, loss factor, loss tangent, waveguide, concrete setting process, RFID, Debye, MSE.

\section{INTRODUCTION}

C ONCRETE is the most widely used material in the world. More than 2 billion tones of this material are produced every year [1]. The embedding of wireless sensors, either to monitor the evolution of the setting process or to detect hazardous conditions in the structure, has been proposed as a solution to guarantee the integrity of concrete structures [2]. Hence, the wireless characterization and monitoring of its properties becomes a topic of great interest.

During the design process of embedded wireless sensors appropriate to work inside concrete structures, it is necessary to have available highly accurate and reliable data of its electromagnetic properties and their evolution during the hardening process. In this paper, we intend to provide such data.

Most of the work conducted to measure the dielectric properties of high permittivity granular materials employs methods that operate on reflection regime. This is the case of [3], where the Open-ended Coaxial Line method is employed to characterize the electromagnetic properties of concrete at microwave frequencies from wet to oven dried status [4],

This manuscript was submitted on July 25, 2019. This work was supported by the Spanish "Comision Interministerial de Ciencia y Tecnologia" (CICYT) under projects TEC2013-47360-C3-1-P, TEC2016-78028-C3-1-P, MDM20160600, and by the "Unidad de Excelencia María de Maeztu", 2017-2020.

G. González-López is a PhD student with the Antenna Laboratory (AntennaLab) research group inside the Signal Theory and Communications Department of the "Universitat Politècnica de Catalunya" (UPC), Barcelona, Spain. (email: giselle.gonzalez@tsc.upc.edu).

Dr. S. Blanch is an associate professor, and Dr. J. Romeu and Dr. L. Jofre are full professors, at UPC and members of the AntennaLab. (email: blanch, romeu, jofre@tsc.upc.edu). or [5], where the permittivity of cement based materials is measured using Time Domain Reflectometry (TDR).

Probe methods such as [3] are quite appealing when performing Non-Destructive Tests (NDT) as they preserve the sample material. Nevertheless, when measuring the dielectric properties of a medium with high permittivity $\left(\varepsilon_{r}\right)$ and losses $(\sigma)$, it is not recommended to solely relay on the measured reflection coefficient $\left(S_{11}\right)$ (as it is done in reflection methods). The inversion process to retrieve the dielectric permittivity is very sensitive to measurement errors. This is why, under these circumstances, a measurement method that operates on transmission/reflection regime (therefore obtaining information from all four scattering parameters) provides higher reliability on the estimation of the dielectric properties of the material, than a method based on a reflection measurement only.

Some of the most common techniques to measure the dielectric properties of materials are studied in [6] and [7], and insights are provided regarding its applicability scenarios. In [8], a measurement set up operating on transmission/reflection regime composed by a series of waveguides is employed to measure the permittivity of granular materials. In [3], [5], [8][15], the dielectric properties of concrete at specific stages of the setting process are reported, but they are either reflection based or do not measure the complete setting process.

Many authors have addressed the problem of measuring the dielectric properties of granular materials such as concrete at microwave frequencies. Nevertheless, the novelty of the approach we propose in this paper consists on employing a transmission/reflection based measurement method, at a frequency band at which the inner dimensions of a waveguide are large enough to host the granular material, that is Debye frequency-extended to surmount the bandwidth limitation of traditional waveguides.

As a consequence of the chemical reactions that take place during the setting process, cement based materials present a very high complex permittivity and electromagnetic properties that change with time. When attempting to design a device to work embedded in a medium with high complex permittivity $\left(\varepsilon_{r}\right)$, there are two paramount elements to be taken into consideration: the attenuation due to the ohmic losses of the material, and the matching of the device [16]. The first one, accounts for the maximum depth where the embedding device can be located. The second one is related to the impedance match of the antenna, which is a quite critical aspect when designing antennas with very small real part impedance and/or highly 
reactive imaginary part, as in the case of RFID applications, sensitive to the dielectric properties of the embedding medium [17].

The exothermic process that happens during the hydration of cement influences the way the temperature and the dielectric properties change throughout the setting process of concrete [18]. From the behaviour of the temperature curves, information regarding the strength and stiffness of freshly placed concrete can be extracted [18]-[20]. Also, the thermal gradient produced during the first 24 hours has great impact on the long term performance of concrete structures [21], [22]. Given the importance of monitoring the internal temperature of concrete, this paper also studies its relation with the complex permittivity.

All the measurements have been conducted for frequencies from $0.7 \mathrm{GHz}$ to $1.1 \mathrm{GHz}$. These results are then frequencyextended up to $3 \mathrm{GHz}$, through a validated Debye model. We have chosen the frequency $0.868 \mathrm{GHz}$ for the results exhibited in Section III as a function of time, for being this a reference frequency in Europe for operating either active or passive devices, and in particular for RFID operation.

With the aforementioned in mind and as an extension of the work conducted in [23], a rectangular waveguide measurement set up has been designed and manufactured to accurately characterize the electromagnetic properties of high permittivity granular materials from $0.7 \mathrm{GHz}$ to $1.1 \mathrm{GHz}$, and their evolution over time. A description of the characteristics of the rectangular waveguide measurement set up, as well as the equations used to extract the permittivity of the medium under test (MUT) from the measured scattering parameters, are included in Section II. Section III contains the experimentally obtained permittivity as a function of time and the Debye frequency-extended estimated complex permittivity. Finally, Section IV provides conclusions regarding the obtained results.

\section{Set Up for the Permittivity Measurement}

In this section, a brief definition of the terminology employed to define the parameters that will further on appear is included. A short explanation of the elements taken into consideration to choose the definitive measurement set up, followed by a description of the set up itself, is also added. Once the main characteristics of the transmission/reflection measurement set up have been displayed, the equations used to extract the permittivity value from the measured parameters are introduced. A short analysis of the layout of the devices employed to measure the internal temperature of the reaction as well as a verification of the accuracy, uncertainty and repeatability of the measurement set up, are included as well. The procedure to frequency-extend the measured complex permittivity through a Debye model is explained and validated at the end of this section.

\section{A. Terminology Definition}

For a general lossy dielectric and time convention $e^{j w t}$ (where $\omega$ is the angular frequency), the relative complex permittivity $\left(\varepsilon_{r}\right)$ is defined as in (1) [24].

$$
\varepsilon_{r}=\varepsilon_{r}^{\prime}-j \varepsilon_{r}^{\prime \prime}
$$

$\varepsilon_{r}^{\prime}$ is defined as the real part of the relative complex permittivity (some authors also define $\varepsilon_{r}^{\prime}$ as the dielectric constant [3]), and $\varepsilon_{r}^{\prime \prime}$ is the imaginary part, which accounts for the loss in the medium [25]. The loss tangent is computed from (2), while the conductivity is extracted from (3) [26].

$$
\begin{aligned}
& \tan \delta=\frac{\varepsilon_{r}^{\prime \prime}}{\varepsilon_{r}^{\prime}} \\
& \sigma=\omega \varepsilon_{0} \varepsilon_{r}^{\prime \prime}
\end{aligned}
$$

The complex propagation constant $(\gamma)$ of a plane wave in a general lossy medium is defined as:

$$
\gamma=\alpha+j \beta
$$

The real and the imaginary parts of (4) are the attenuation constant $(\alpha)$ and the phase constant $(\beta)$, respectively.

The propagation factor $(\mathrm{P})$ for a wave propagating througha layer of length $d$ of the material is defined in [24], [27]:

$$
\begin{aligned}
P & =e^{-\gamma d} \\
& =e^{-\alpha d} e^{-j \beta d}
\end{aligned}
$$

The attenuation constant $(\alpha)$ can be related to the dielectric properties of the medium by means of the expressions defined in [27], [28], expressed in terms of $w$, the velocity of light in vacuum $\left(c_{0}\right)$ and the relative complex permittivity $\left(\varepsilon_{r}\right)$ in $(1)$ :

$$
\alpha=\frac{w}{c_{0}} \sqrt{\varepsilon_{r}^{\prime}}\left\{\frac{1}{2}\left[\sqrt{1+\left(\frac{\varepsilon_{r}^{\prime \prime}}{\varepsilon_{r}^{\prime}}\right)^{2}}-1\right]\right\}^{1 / 2}
$$

from which the attenuation constant in $\mathrm{dB} / \mathrm{cm}$ results on (7):

$$
\alpha(d B / c m)=20 \log e^{-\alpha 0.01}
$$

\section{B. Design Considerations}

The most often used transmission lines for material characterization are either coaxial structures, strip lines or waveguides [8], [13], [29]. However, the first two require small size samples, which is a drawback when it comes to measuring granular materials. On the other hand, a rectangular waveguide propagating the fundamental $\mathrm{TE}_{10}$ mode at the $800-900$ $\mathrm{MHz}$ band of interest, has a cross-section (a, Figure 3 ) of the order of tens of centimeters which is suitable to be filled with granular materials such as concrete or mortar.

\section{Description of the Waveguide Structure}

Waveguide measurement systems are appropriate to measure different kind of materials and in particular semi-solid or solid structures, which are cut to fit inside the guide. Figures 1 to 3 show all the parts that compose the manufactured measurement set up. The section of the waveguide filled with the material will be refereed to as the "Sample holder". It has an inner length $(d)$ of $300 \mathrm{~mm}$, inner width $(a)$ of $262 \mathrm{~mm}$ and inner height (b) of $117 \mathrm{~mm}$. 


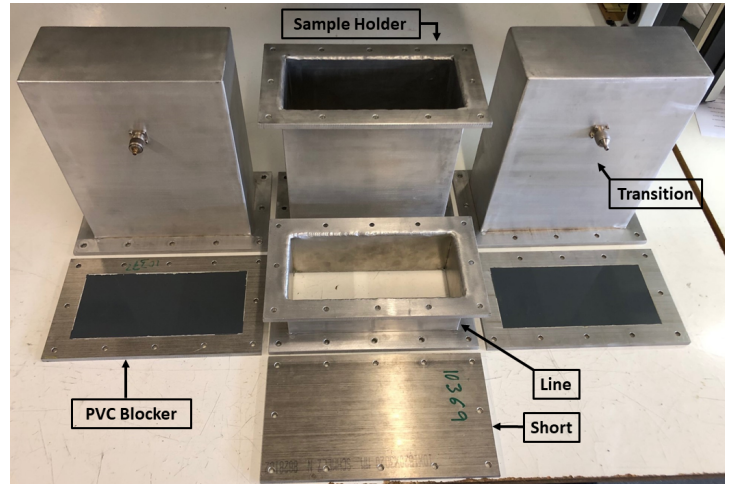

Fig. 1: Wave-Guide measurement Set Up.

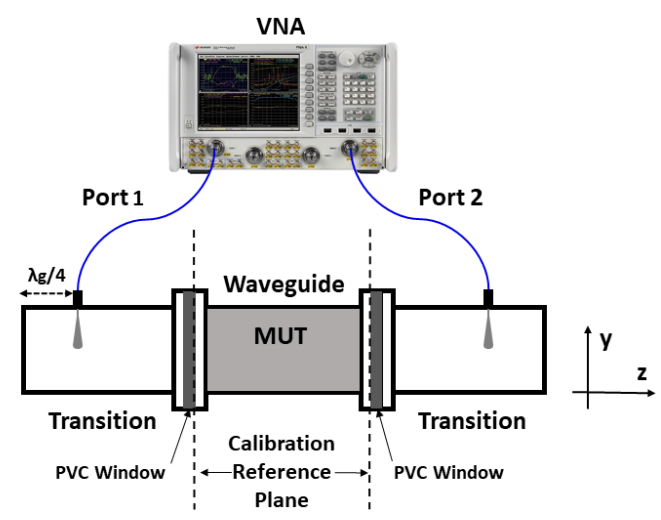

Fig. 2: Schematic of the Measurement Set Up.

In this case, the material under test has a rather liquid consistency in an early stage, so two "PVC blockers" will be placed on the sides of the "Sample holder" to prevent any leakage. These "PVC blockers" are $5 \mathrm{~mm}$ thick and its effect is removed during the calibration. Two coaxial-waveguide "Transitions" are to be placed on each side of the guide, each one connected to one port of a Vector Network Analyzer (VNA) through coaxial cables with $\mathrm{N}$-type connectors. The VNA N5247A from Agilent Technologies has been used to conduct this measurement. To conduct the TRL (Through Reflect Line) calibration, a piece shaped as a "Shorted" section and a "Line" section have also been manufactured. Figure 2 describes the schematic of the whole measurement set up.

To improve the broadband characteristics, the section of the inner conductor of the coaxial feed inserted into the rectangular guide is widened with a conical monopole with hemispherical end (Figure 3). This monopole has been attached to the coaxial using a conductive epoxy.

\section{Retrieving the Relative Complex Permittivity $\left(\varepsilon_{r}\right)$ from measured S-parameters}

The value of the permittivity of the material is extracted from the S parameter measurement of the "Sample Holder" section filled with the material under test (MUT).

From the measured scattering parameters ([S]), the transmission ([T]) and reflection $([\Gamma])$ coefficients are obtained through equations (8) to (10), applying the Nicolson-Ross-Weir model

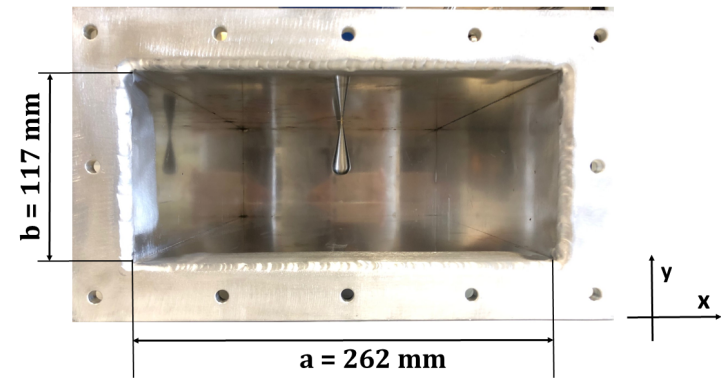

Fig. 3: Coaxial-waveguide Transition.

in [30] for the calculation of the complex dielectric permittivity at microwave frequencies. Where $\mathrm{A}$, is an intermediate variable defined for simplification purposes.

$$
\begin{gathered}
T=\frac{S_{11}+S_{21}-\Gamma}{1-\left(S_{11}+S_{21}\right) \Gamma} \\
\Gamma=A \pm \sqrt{A^{2}-1} \\
A=\frac{S_{11}{ }^{2}-S_{21}{ }^{2}+1}{2 S_{11}}
\end{gathered}
$$

The transmission parameter ([T]) for a wave propagating through the sample is equal to the propagation factor in (5), corresponding $d$ in this case to the length of the "Sample holder" section of the waveguide. The complex propagation constant $(\gamma)$ for the $\mathrm{TE}_{10}$ mode (dominant mode) in a rectangular waveguide filled with a dielectric is given in (11), being $\varepsilon_{r}$ the parameter to be calculated [24].

$$
\gamma=\sqrt{\left(\frac{\pi}{a}\right)^{2}-\left(\frac{w}{c_{0}}\right)^{2} \varepsilon_{r}}
$$

\section{E. Temperature Monitoring Set Up}

In order to monitor the evolution of the internal temperature of the MUT during its setting process, several type K thermocouples connected to a data logger have been employed [31].

It is of interest to measure the internal temperature of the material at different depths (in the $\mathrm{x}$ axis) because the temperature inside the structure is dependent on the distance to the center, where it is expected to detect the highest temperature record due to the low thermal conductivity of the material.

In this experiment, four thermocouples have been inserted into the waveguide at different heights ( $\mathrm{z}$ axis) and depths ( $\mathrm{x}$ axis), through small holes drilled on its side, placed so they would be perpendicular to the direction of the E-field inside the guide, and therefore its effect could be dismissed. To validate this statement, a measurement of the permittivity of the empty waveguide, with and without the thermocouple cables, was conducted (Section II-G).

Two extra thermocouples have been placed outside the waveguide to measure room temperature. In this way, it would be possible to compare the internal and the external temperature, and to detect when the internal temperature of the reaction stabilizes approaching room temperature. 


\section{F. Uncertainty Analysis}

The main sources of error in a transmission/reflection measurement have been evaluated in [32]. These sources include (a) errors in the measurement of the scattering parameters, (b) air gaps between the sample and the sample holder, (c) uncertainty in the dimensions of the sample holder, (d) uncertainty in the sample length, (e) uncertainty in the position of the reference plane, and (f) uncertainty in the exact position of the sample inside the sample holder.

Some of these sources of error have been either mitigated or removed by taking specific considerations at the manufacturing and measuring stages. The air gaps between the sample and the PVC blockers are corrected by totally filling the sample holder and tightening the PVC blocker on top. Appropriate mechanical tolerances in the manufacturing of the measurement set up aid mitigating the uncertainty in the dimensions of the sample holder. As long samples are proven to reduce the measurement uncertainty [33], and in this case the sample fills the entire sample holder, and the PVC blockers at each side of the concrete sample are included during the TRL calibration, it is possible to abridge the uncertainty arising from the position of the sample and from the reference plane respectively.

Hence, the main sources of measurement uncertainty in this specific set up will be more likely associated to the scattering parameters measurement and to the sample length. The estimated combined uncertainty in the measured relative permittivity obtained from the $1.0 \%$ to $2.0 \%$ scattering parameters' measurement uncertainty provided in the datasheet of the N5247A VNA, and an assumed sample length uncertainty of $0.5 \%$ (1.5 mm over $300 \mathrm{~mm})$, results on a figure below $4 \%$ for both the real and the imaginary part, which is in agreement with the results provided in [32].

\section{G. Validation of System Accuracy and Repeatability}

As a mean to asses the accuracy of the chosen measurement set up, we have decided to measure a well-characterized high permittivity lossy material. The selected material is isopropyl alcohol (also known as isopropanol or propan-2-ol), and its complex permittivity has been characterized in [34] as a function of frequency and temperature. Figure 4 compares the real and imaginary part of the relative permittivity $\left(\varepsilon_{r}\right)$ of isopropanol measured at $24.6^{\circ} \mathrm{C}$ (room temperature), with the values extracted from [34] for the same temperature. The estimated accuracy of the results exhibited in Figure 4, averaged throughout the displayed frequency band is of $0.2 \%$, with a maximum error of $1.1 \%$ (for both real and imaginary parts) at $1.09 \mathrm{GHz}$. This is in agreement with the previous uncertainty analysis.

To validate the repeatability of the chosen measurement set up, Figure 5 displays the measured permittivity of the empty waveguide before the measurement and once the sample had been removed ( 9 days later). It is important to notice the excellent repeatability of the results, given the fact that the whole measurement set up had to be disassembled and assembled back together between measurements. The repeatability is within $0.5 \%$ in both the real and the imaginary part of the relative complex permittivity.

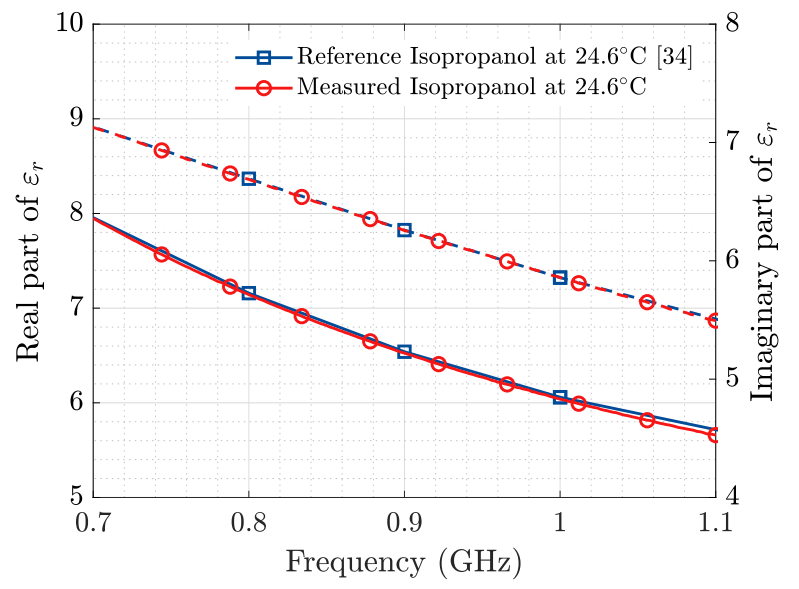

Fig. 4: Measured Permittivity of Isopropanol compared to reference values provided in [34]. Real part represented as straight lines and imaginary part as dashed lines.

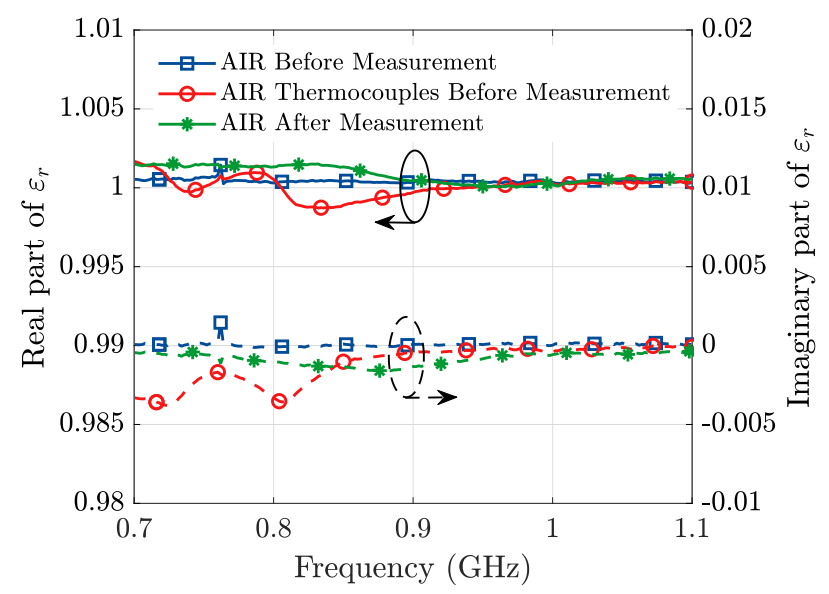

Fig. 5: Air permittivity measurement. Real part represented as straight lines and imaginary part as dashed lines.

A measurement of the empty waveguide with the thermocouples placed at their designated positions is also included in Figure 5. The dispersion introduced in the measurement by their presence is also under $0.5 \%$ for the real and the imaginary part of the relative permittivity, therefore verifying that its effect can be dismissed.

\section{H. Debye frequency extension}

The manufactured waveguide has an operation bandwidth spanning from 0.7 to $1.1 \mathrm{GHz}$. To frequency-extend the results provided for the complex permittivity of the measured materials, we have employed the Debye relaxation model in [35] as a mean to capture their experimental behaviour.

It is known from [36], [37] that the Debye- $\Gamma$ model offers an appropriate representation of the behaviour of the relative permittivity of concrete over the frequency interval of interest. In [36], the frequency response of a concrete sample is measured up to $20 \mathrm{GHz}$ and a second relaxation frequency around $10 \mathrm{GHz}$ is observed. Therefore, it is possible to state that this 


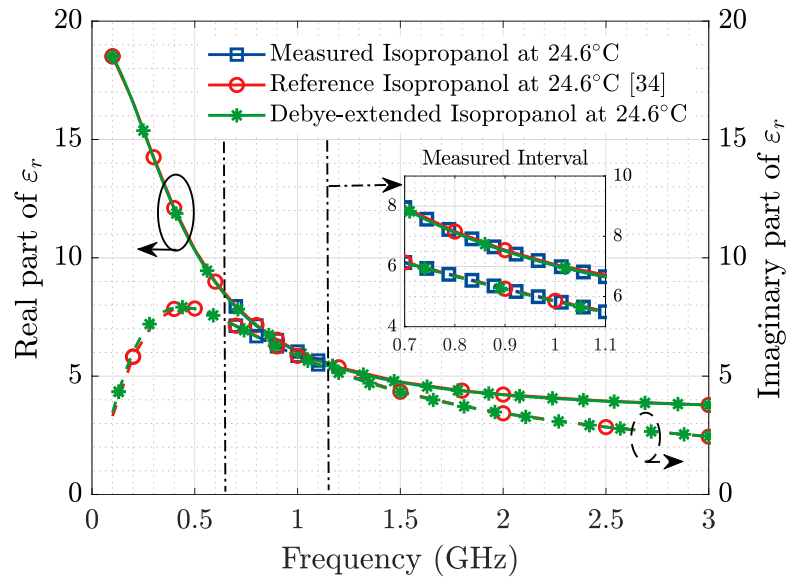

Fig. 6: Debye frequency-extended permittivity of isopropanol compared to reference values provided in [34]. Real part represented as straight lines and imaginary part as dashed lines. The inset corresponds to measured values.

model provides a good level of accuracy for frequencies up to $3.3 \mathrm{GHz}$, as this frequency is under one third of the second relaxation frequency of concrete.

To assess the expected accuracy of the Debye model on the estimation of the dielectric properties of high permittivity lossy materials (such as concrete) at frequencies above $1.1 \mathrm{GHz}$ and up to $3.3 \mathrm{GHz}$, we have extracted the relaxation parameters of the measured sample of isopropanol, and frequency-extended them in the interval of interest. We have used the expression for the Debye- $\Gamma$ model in (12) which provides a good representation of the behaviour of the complex permittivity of this type of materials [34]. The parameters $\varepsilon_{\infty}$ (high frequency permittivity limit), $\varepsilon_{s}$ (static permittivity), $f_{r}$ (relaxation frequency), $\sigma$ (static conductivity) and $\Gamma$ (fitting parameter) have been obtained through minimum squared error (MSE) estimation. The third term of equation (12) naturally models the loss of the dielectric material [38], while the positive quantity $(\Gamma)$ in the last term represents the tail of the second relaxation frequency [34].

$$
\varepsilon_{r}=\varepsilon_{\infty}+\frac{\varepsilon_{s}-\varepsilon_{\infty}}{1+j \frac{f}{f_{r}}}-j \frac{\sigma}{\omega}-j f \Gamma
$$

The resulting permittivity of isopropanol has been compared to the data measured with the manufactured waveguide and to the reference data extracted from [34] as depicted in Figure 6 . According to these results, the Debye- $\Gamma$ model is capable to estimate the complex permittivity of the material with an accuracy of $0.45 \%$ for the real part of the permittivity and $0.56 \%$ for the imaginary part (averaged over the displayed frequency band). The maximum error in the estimation is of $0.52 \%$ for the real part at $0.45 \mathrm{GHz}$ and of $0.8 \%$ for the imaginary part at $0.15 \mathrm{GHz}$.

\section{Measured Electromagnetic Properties of CONCRETE}

The results included in this section have been obtained for two samples of H-25 PRO concrete measured during different seasons (winter and spring) and a sample of self-levelling mortar. Each sample has a volume of $9.2 \mathrm{dm}^{3}$ and has been prepared using the ratios in Table I. The concrete samples have been monitored during 9 days, while the sample of mortar has been monitored during 6 days. Measurements were taken every 5 minutes in every case. The results shown in this section as a function of time have been plotted at $0.868 \mathrm{GHz}$. The measurements started 20 minutes after preparing the mixture, in average. Figure 7 illustrates the whole configuration of an ongoing measurement.

The relative complex permittivity depicted in Figure 8 has been extracted from (11). The loss tangent $(\tan \delta)$ and the conductivity $(\sigma)$ (Figure 10) have been computed from (2) and (3), respectively. While the attenuation factor $(\alpha(\mathrm{dB} / \mathrm{cm}))$ in Figure 11, has been obtained from (7).

At the beginning of the reaction produced during the setting process of cement based materials, there is a high water concentration. This, along with the inherent characteristics of the material, generates a high relative permittivity at early

TABLE I: Sample Preparation Ratios of H-25 PRO concrete and self-levelling mortar. These figures have been computed in accordance with the data provided by the manufacturers and the EHE-08 instruction [39]. The volume of the sample is of $9.2 \mathrm{dm}^{3}$ and $\phi$ is the diameter of the aggregates.

\begin{tabular}{c|c|c}
\hline Water & $\begin{array}{c}\text { H-25 PRO } \\
\text { concrete }\end{array}$ & $\begin{array}{c}\text { Self-levelling } \\
\text { mortar }\end{array}$ \\
\hline Cement & $195 \mathrm{l} / \mathrm{m}^{3}$ & $522 \mathrm{l} / \mathrm{m}^{3}$ \\
\hline Sand $(\phi=0-4 \mathrm{~mm})$ & $325 \mathrm{~kg} / \mathrm{m}^{3}$ & $325 \mathrm{~kg} / \mathrm{m}^{3}$ \\
\hline Gravel $(\phi=4-10 \mathrm{~mm})$ & $979 \mathrm{~kg} / \mathrm{m}^{3}$ & $1153 \mathrm{~kg} / \mathrm{m}^{3}$ \\
\hline
\end{tabular}

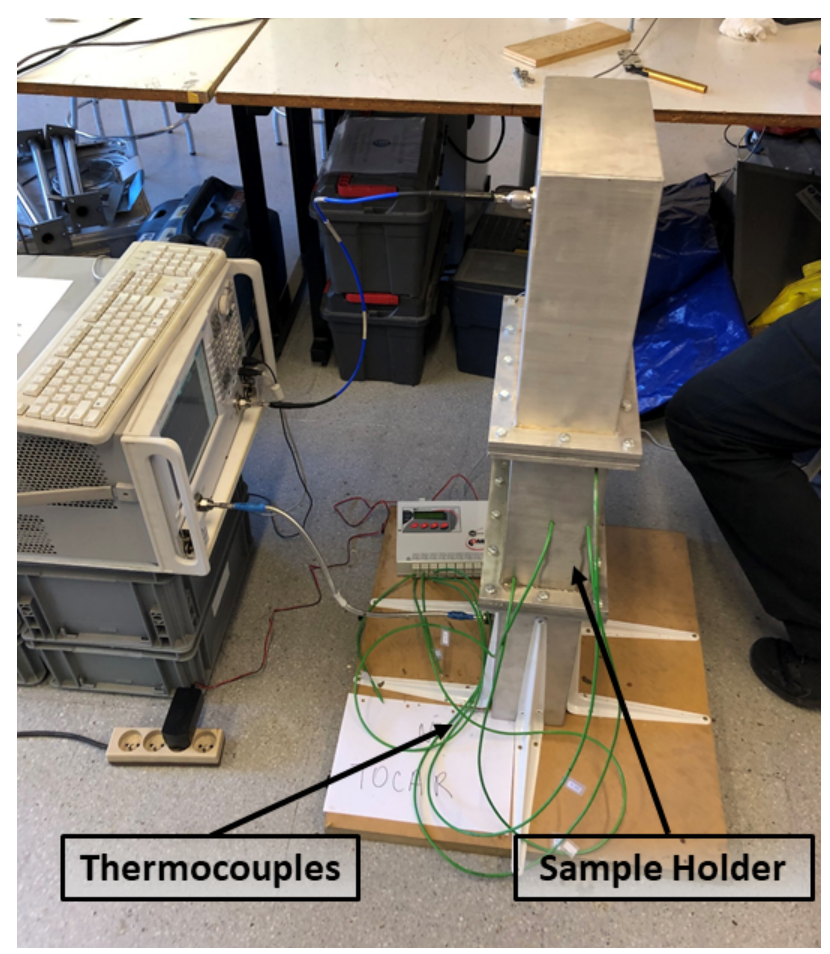

Fig. 7: Set Up of the ongoing Measurement. 


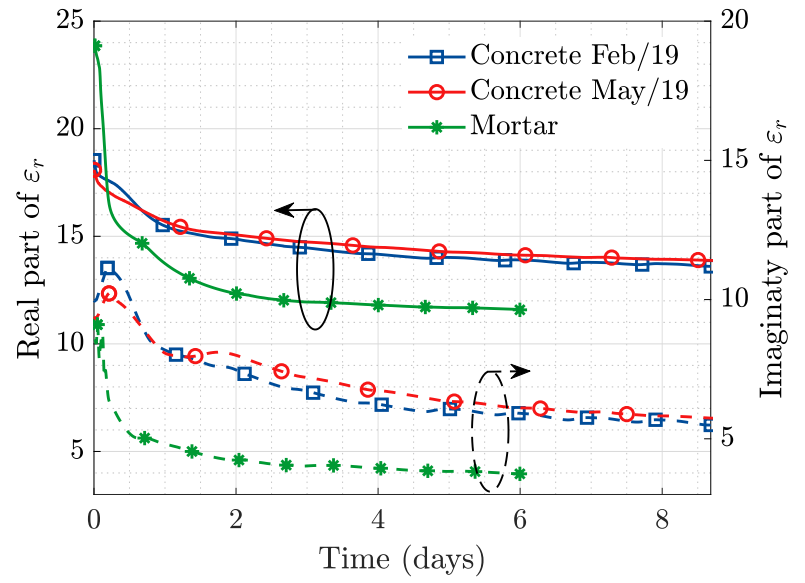

Fig. 8: Permittivity evolution over time of measured materials at $0.868 \mathrm{GHz}$.
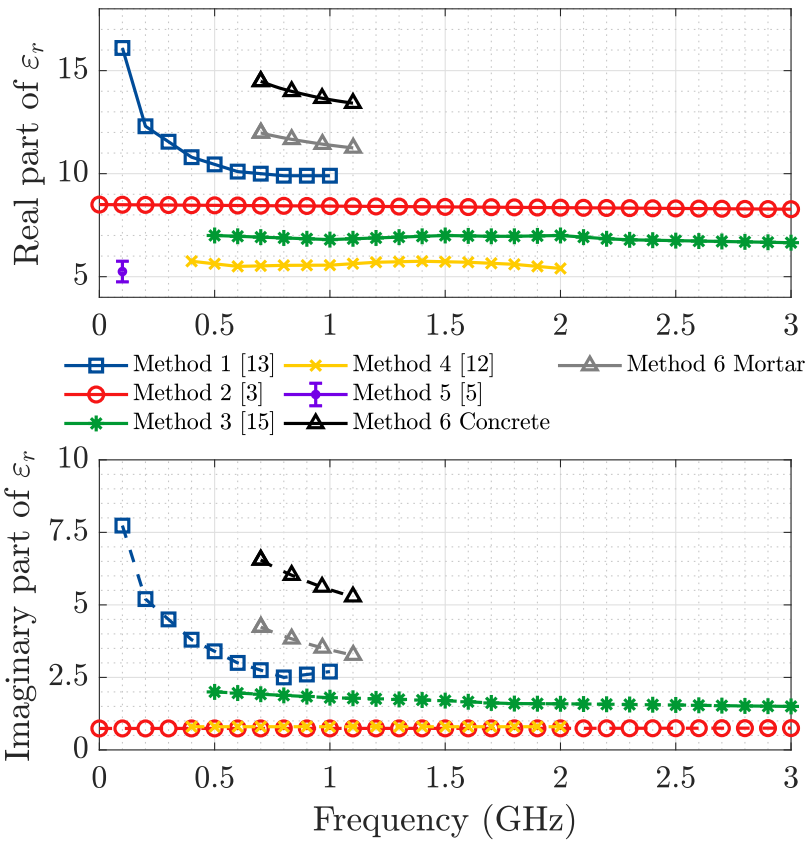

Fig. 9: Published values of concrete permittivity obtained through measurement methods in Table II. These data have been collected (within feasibility) for concrete samples in a stable stage of the setting process. Values measured for selflevelling mortar have also been included.

stages of the process as it can be appreciated from Figure 8. It is also possible to notice that both the real and the imaginary parts of the relative permittivity tend to decrease as the reaction stabilizes. While the real part of the permittivity of concrete lowers down from 18.5 to 13.6 , the imaginary part goes from a maximum of 11.2 to a final value around 5.0. For the case of mortar, the initial maximum of 23.9 in the real part of $\varepsilon_{r}$ stabilizes around 11.0 after 3 days, and the imaginary part goes from 9.1 to 4.0 in the same amount of time.

It is of interest to call attention on the peak produced in the dielectric properties during the first 24 hours of the reaction. During this time lapse, an abrupt increase in the losses of the
TABLE II: Published Concrete Permittivity Measurement Methods.

\begin{tabular}{l|l|l}
\hline & Method & $\begin{array}{l}\text { Operation } \\
\text { Regime }\end{array}$ \\
\hline Method 1 & Coaxial Closed Cell [13] & $\mathrm{Tx} / \mathrm{Rx}$ \\
Method 2 & Open-ended Coaxial Probe [3] & $\mathrm{Rx}$ \\
Method 3 & Free Space Measurement [15] & $\mathrm{Tx} / \mathrm{Rx}$ \\
Method 4 & Ground Penetrating Radar (GPR) [12] & $\mathrm{Rx}$ \\
Method 5 & Time Domain Reflectometry (TDR) [5] & $\mathrm{Rx}$ \\
Method 6 & Waveguide Transmission Line (this paper) & $\mathrm{Tx} / \mathrm{Rx}$ \\
\hline
\end{tabular}

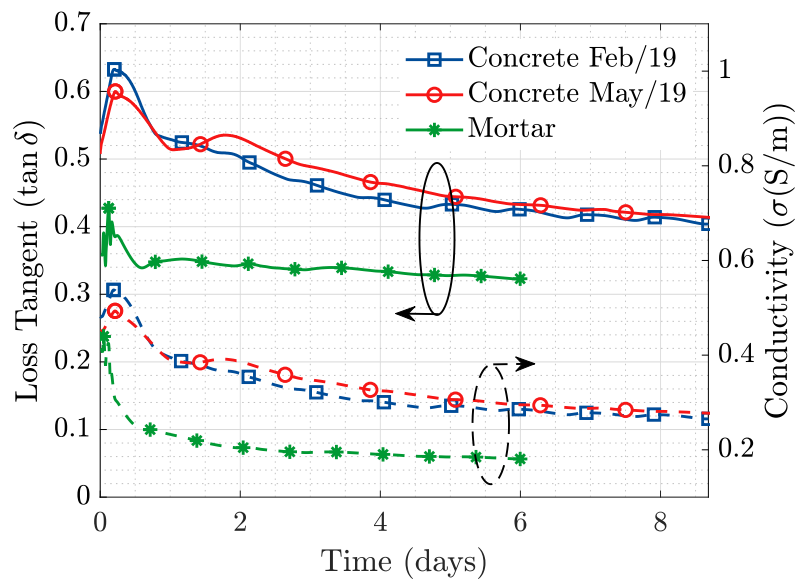

Fig. 10: Loss tangent and conductivity $(\mathrm{S} / \mathrm{m})$ evolution over time of measured materials at $0.868 \mathrm{GHz}$.

materials take place, followed by a quite sharp decrease that starts stabilizing by the end of the first day of measurement. This interval coincides with the most critical moment of the chemical reaction of hydration of cement.

The relative complex permittivity values obtained with this measurement are higher (around $45 \%$ for the real part of the relative permittivity and $80 \%$ for the imaginary part) than those reported in [3], [5], [8]-[15]. Figure 9 compares the values obtained in this paper for the permittivity of concrete, with previously published results of concrete permittivity obtained through different measurement methods (Table II) either transmission/reflection based, or only reflection based. Data for Time Domain Reflectometry (TDR) (Method 5) has been extracted from [5] and frequency converted applying the expressions in [40]. The results provided in this paper are proof of the convenience of employing waveguide structures to measure high permittivity lossy granular materials. They provide an accurate representation of the variations produced in the electromagnetic properties of concrete during its setting process.

The loss tangent and the conductivity (Figure 10) exhibit the same behaviour than the one seen in the imaginary part of the relative permittivity. This is an expected behaviour as these parameters are an expression of the losses present in the material. In this case, $\tan \delta$ lowers from a maximum above 0.6 or concrete, produced at the beginning of the reaction, to a value around 0.4 where it shows a more stable behaviour. Self-levelling mortar, on the other hand, exhibits fewer losses within 0.3 and 0.4 . 


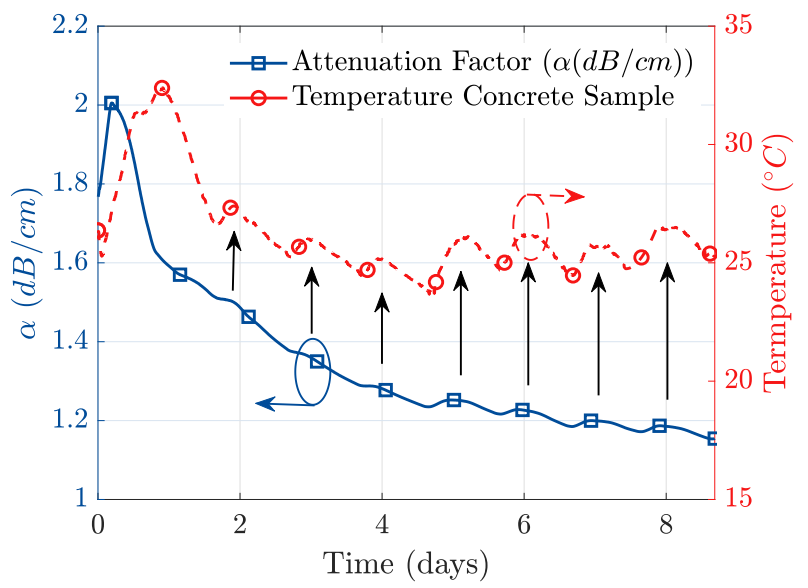

Fig. 11: Attenuation Factor at $0.868 \mathrm{GHz}$ and Temperature Correlation of concrete over Time.

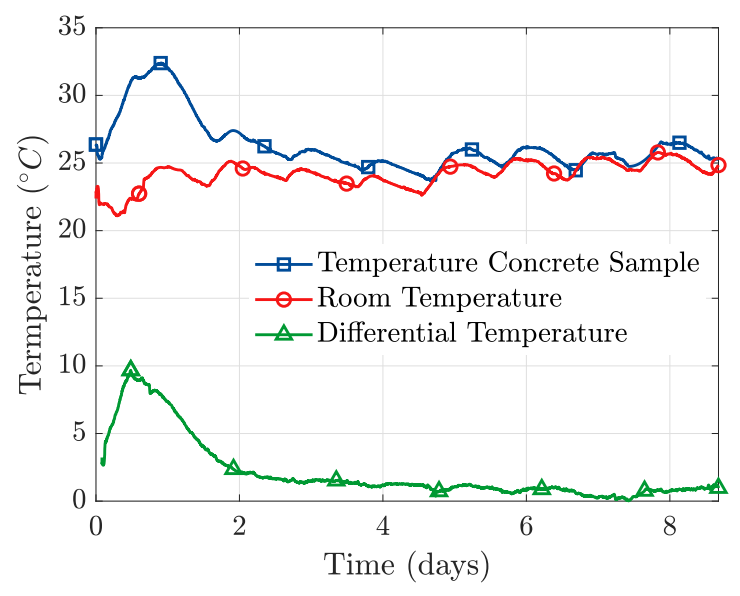

Fig. 12: Differential Temperature.

The attenuation factor $(\alpha)$ inside concrete expressed in $\mathrm{dB} / \mathrm{cm}$ is illustrated in the left vertical axis of Figure 11. During the first 24 hours of the setting process, the recorded attenuation level inside concrete reaches a peak of $2 \mathrm{~dB} / \mathrm{cm}$. After the initial peak, $\alpha$ starts slowly stabilizing remaining around $1.2 \mathrm{~dB} / \mathrm{cm}$, value reached after 4 days of measurements.

After the first day of setting, the presence of fluctuations with a 24 hour period due to a "night and day temperature effect" can be easily distinguished (Figure 11). These variations are due to the temperature changes in the laboratory. In the same figure, the temperature at the center of the concrete sample has been included on the right side of the vertical axis. It is possible to notice that the time instant where the temperature maximums inside concrete are produced, approximately matches the moment when there is also a small maximum in the attenuation factor $(\alpha)$ in the material. In consequence, we attribute the small daily fluctuations in the attenuation factor to the temperature induced variations in the complex permittivity.

In order to remove the influence of the external temperature changes from the temperature evolution of concrete due to the internal chemical reaction alone, the following proce-

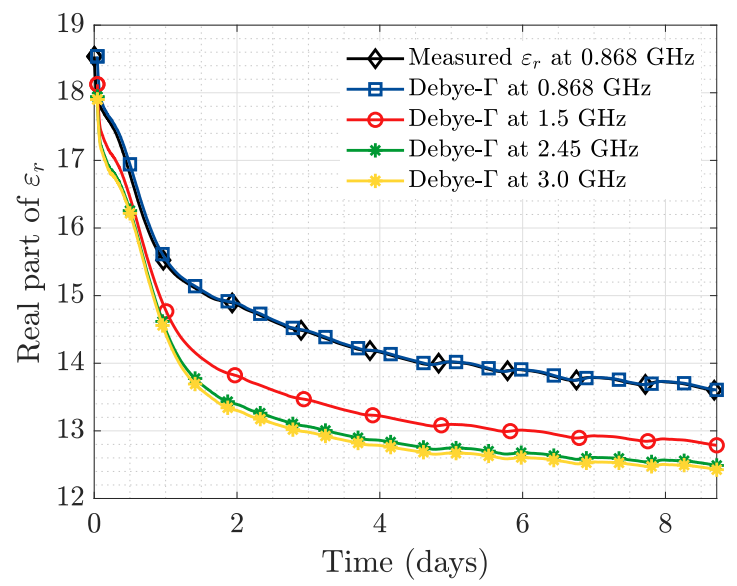

Fig. 13: Debye frequency-extended permittivity of H-25PRO concrete sample. Real Part of $\varepsilon_{r}$.

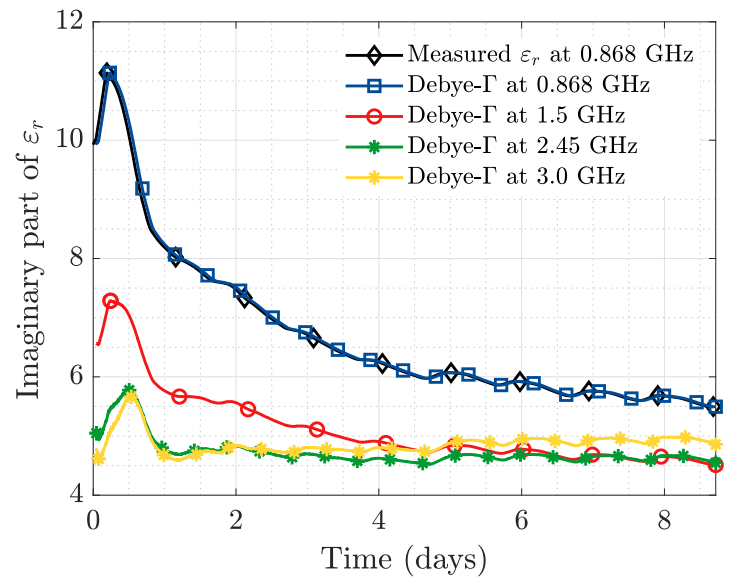

Fig. 14: Debye frequency-extended permittivity of H-25PRO concrete sample. Imaginary part of $\varepsilon_{r}$.

dure has been conducted. Figure 12 depicts the measurement recorded by the thermocouple sensor located at the center of the concrete sample, and the one recorded by one of the thermocouples measuring room temperature. A time shift of approximately 2 hours can be easily noticed between these two plots, which is induced by the time the external temperature takes to influence the temperature inside concrete due to the low thermal conductivity of this material. This day and night temperature effect is corrected taking into account the aforementioned 2 hour time shift, and the obtained "differential temperature" presents an almost stable behaviour and a decreasing monotony that stabilizes for values close to " 0 ". This shows that after the 9 day period, the setting process is almost completed. This information is important because the temperature evolution of concrete is an indicator of the stage of the internal chemical reactions, and it can be used in future work to relate the complex permittivity values to the specific stages of the setting process. 
TABLE III: Debye- $\Gamma$ model parameters for the relative complex permittivity of H25-PRO concrete sample 1 .

\begin{tabular}{c|c|c|c|c}
\hline Parameter & Day 1 & Day 2 & Day 4 & Day 8 \\
\hline$\varepsilon_{\infty}$ & 14.3035 & 13.1099 & 12.6258 & 12.3679 \\
$\varepsilon_{s}$ & 19.9943 & 20.7391 & 20.6300 & 20.6215 \\
$f_{r}$ & 0.4558 & 0.4776 & 0.4253 & 0.3855 \\
$\sigma$ & 0.2520 & 0.1705 & 0.1068 & 0.0772 \\
$\Gamma$ & 0.7733 & 0.8750 & 1.0167 & 1.1576 \\
\hline
\end{tabular}

TABLE IV: Propagation Loss of a wireless sensor operating at $0.868 \mathrm{GHz}$ embedded in a structure of H-25 PRO concrete.

\begin{tabular}{c|c|c|c}
\hline & Time & $\varepsilon_{r}$ & $P L(d B)$ \\
\hline$d=0.1 m$ & 0.5 & $16.8-j 10$ & 54.5 \\
& 4 & $14.2-j 6.2$ & 45 \\
& 8 & $13.7-j 5.7$ & 40.9 \\
\hline$d=0.2 m$ & 0.5 & $16.8-j 10$ & 73.7 \\
& 4 & $14.2-j 6.2$ & 57.5 \\
& 8 & $13.7-j 5.7$ & 50.6 \\
\hline
\end{tabular}

\section{A. Debye frequency-extended Permittivity of Concrete}

The values measured for the complex permittivity of the first sample of concrete from 0.7 to $1.1 \mathrm{GHz}$ have been frequencyextended applying the fitted Debye model in (12), having an in-band accuracy below $0.1 \%$ for both real and imaginary part.

Figures 13 and 14 illustrate the measured permittivity of the first sample of concrete as a function of time at 0.868 $\mathrm{GHz}$, compared to the complex permittivity values obtained at $1.5,2.45$ and $3.0 \mathrm{GHz}$ for the fitted Debye model. The fitting parameters obtained for the Debye- $\Gamma$ model at different time instants are included in Table III, and they can eventually be extended to other frequencies. From Table III it is possible to notice that the most significant variation is produced in the conductivity $(\sigma)$, which agrees with the results presented in [41].

For a scenario involving a wireless sensor operating at 0.868 $\mathrm{GHz}$, embedded at a certain depth $d_{\text {con }}$ (distance from the interface air-medium to the sensor) inside a concrete structure, and an external reader placed at a distance $d_{\text {air }}$ (distance from the interface to the reader), the Propagation Loss (PL) in dB (considering both ohmic and propagation losses) between the sensor and the reader (obtained through full wave simulations in HFSS) at different stages of the setting process, for $d_{c o n}$ $=0.1 \mathrm{~m}$ and $0.2 \mathrm{~m}$ and a reading distance $d_{a i r}=0.3 \mathrm{~m}$, is as depicted in Table IV. A $0 \mathrm{~dB}$ gain has been considered for the antennas of the reader and the sensor.

It can be extracted from Table IV that the propagation loss (PL) may vary from 54.5 to $73.7 \mathrm{~dB}$, measured half a day after the start of the setting process. Eight days later, this values reduce to a 40.9 to $50.6 \mathrm{~dB}$ range of propagation loss, that may no longer exceed the $60 \mathrm{~dB}$ threshold of most passive RFID systems [42]. These figures may provide clear guidance for the design of wireless systems to operate with sensors embedded in concrete, and for the selection of actual operating frequency or feeding mechanism (active or passive RFID) of the sensor.

\section{CONCLusions}

The waveguide transmission line set up has proven to be an accurate method to characterize the evolution of the microwave properties of high permittivity granular materials.
This opens the door to the possibility of employing this kind of measurement set up for the characterization of media with high losses and a time changing behaviour.

It is possible to apply relaxation models such as the Debye model to frequency-extend the complex permittivity measurement of granular materials such as concrete or mortar in scenarios where it is not feasible to conduct a measurement at higher frequencies. This happens for example when the maximum sample size required by the set up is smaller than the minimum feasible sample size of the material under test.

The hydration reactions that take place inside concrete or mortar during the first 24 hours of the setting process, along with the high concentration of water present in the mixture at this stage, contribute to the high valued relative complex permittivity $\left(\varepsilon_{r}\right)$ detected at this moment of the reaction.

The electromagnetic properties of concrete exhibit a stable behaviour after the first week of the setting process. Nevertheless, after this amount of time, it is still required to overcome $18 \mathrm{~dB}$ in ohmic losses attenuation only, to communicate with a device embedded $15 \mathrm{~cm}$ deep, while it requires $30 \mathrm{~dB}$ at the time instant when the attenuation peak is produced.

The temperature of the external medium surrounding the concrete sample has certain influence over the temperature of concrete, and therefore over its electromagnetic properties, which fluctuate following the day and night temperature variation.

These results are of great interest for wireless applications (such as RFID based sensing) involving devices embedded in concrete operating at $868 \mathrm{MHz}$, or other of the analysed frequencies. These data can be employed to determine either penetration depths or matching of the device under the effect of the dielectric properties of the surrounding medium. Furthermore, for those applications where it is a must being able to sustain a reliable and stable communication link with an embedded device since the beginning of the setting process of concrete, it is paramount to know the exact variations that take place on its relative complex permittivity, either to ensure good matching or to be able to adjust the transmission equation so it is possible to withstand the peak produced in the ohmic loss attenuation during the first stages of the process.

The results provided in this manuscript can be used to extend the analysis conducted in Table IV at $0.868 \mathrm{GHz}$, to 1.5 , 2.45 and $3.0 \mathrm{GHz}$, and to other stages of the setting process of concrete.

\section{ACKNOWLEDGMENT}

We would like to thank the faculty of civil engineering from UPC for preparing the concrete and the self-levelling mortar samples measured in this paper, and for their insights on the chemical reaction of hydration of concrete seen from the perspective of a civil engineer.

\section{REFERENCES}

[1] J. Mitchell Crow, "The concrete conundrum," Chemistry World, pp. 6266, 2008. 
[2] J. T. Simonen, M. M. Andringa, K. M. Grizzle, S. L. Wood, and D. P. Neikirk, "Wireless sensors for monitoring corrosion in reinforced concrete members," in Smart Structures and Materials 2004: Sensors and Smart Structures Technologies for Civil, Mechanical, and Aerospace Systems, vol. 5391. International Society for Optics and Photonics, 2004, pp. 587-597.

[3] H. C. Rhim and O. Buyukozturk, "Electromagnetic properties of concrete at microwave frequency range," Materials Journal, vol. 95, no. 3, pp. 262-271, 1998.

[4] J. S. Bobowski and T. Johnson, "Permittivity measurements of biological samples by an open-ended coaxial line," Progress In Electromagnetics Research, vol. 40, pp. 159-183, 2012.

[5] A. Cataldo, E. De Benedetto, and G. Cannazza, "Hydration monitoring and moisture control of cement-based samples through embedded wirelike sensing elements," IEEE Sensors Journal, vol. 15, no. 2, pp. 1208$1215,2015$.

[6] A. Technologies, "Agilent basics of measuring the dielectric properties of materials. application note," 2006.

[7] C. Kuek, "Measurement of dielectric material properties: application note," Rohde \& Schwarz Regional Headquarters Pte. Ltd.: Singapore, 2012.

[8] E. Piuzzi, G. Cannazza, A. Cataldo, S. Chicarella, E. De Benedetto, F. Frezza, S. Pisa, S. Prontera, and F. Timpani, "Measurement system for evaluating dielectric permittivity of granular materials in the 1.72.6-ghz band," IEEE Transactions on Instrumentation and Measurement, vol. 65, no. 5, pp. 1051-1059, 2016.

[9] Y. Shen, C. L. Law, and W. Dou, "Ultra-wideband measurement of the dielectric constant and loss tangent of concrete slabs," in 2008 ChinaJapan Joint Microwave Conference. IEEE, 2008, pp. 537-540.

[10] M.-K. Olkkonen, V. Mikhnev, and E. Huuskonen-Snicker, "Complex permittivity of concrete in the frequency range 0.8 to $12 \mathrm{ghz}$," in 2013 7th European Conference on Antennas and Propagation (EuCAP). IEEE, 2013, pp. 3319-3321.

[11] B. Filali, F. Boone, J. Rhazi, and G. Ballivy, "Design and calibration of a large open-ended coaxial probe for the measurement of the dielectric properties of concrete," IEEE Transactions on Microwave Theory and Techniques, vol. 56, no. 10, pp. 2322-2328, 2008.

[12] A. Ihamouten, G. Villain, and X. Derobert, "Complex permittivity frequency variations from multioffset gpr data: Hydraulic concrete characterization," IEEE Transactions on Instrumentation and Measurement, vol. 61, no. 6, pp. 1636-1648, 2012.

[13] A. Robert, "Dielectric permittivity of concrete between $50 \mathrm{mhz}$ and 1 ghz and gpr measurements for building materials evaluation," Journal of Applied Geophysics, vol. 40, no. 1-3, pp. 89-94, 1998.

[14] S. Pisa, E. Pittella, E. Piuzzi, P. D'Atanasio, and A. Zambotti, "Permittivity measurement on construction materials through free space method," in 2017 IEEE International Instrumentation and Measurement Technology Conference (I2MTC). IEEE, 2017, pp. 1-4.

[15] B. Davis, C. Grosvenor, R. Johnk, D. Novotny, J. Baker-Jarvis, and M. Janezic, "Complex permittivity of planar building materials measured with an ultra-wideband free-field antenna measurement system," Journal of research of the National Institute of Standards and Technology, vol. 112, no. 1, p. 67, 2007.

[16] C. A. Balanis, Antenna theory: analysis and design. John wiley \& sons, 2016.

[17] A. K. Skrivervik, M. Bosiljevac, and Z. Sipus, "Fundamental limits for implanted antennas: Maximum power density reaching free space," IEEE Transactions on Antennas and Propagation, 2019.

[18] C. Wang and W. Dilger, "Prediction of temperature distribution in hardening concrete," in Proceedings of the International RILEM Conference on Thermal cracking in concrete at early ages, 1995, pp. 21-28.

[19] A. K. Schindler, T. Dossey, and B. F. McCullough, "Temperature control during construction to improve the long term performance of portland cement concrete pavements," Tech. Rep., 2002.

[20] A. M. Neville, Properties of concrete. Longman London, 1995, vol. 4.

[21] H. Yu, L. Khazanovich, M. Darter, and A. Ardani, "Analysis of concrete pavement responses to temperature and wheel loads measured from intrumented slabs," Transportation Research Record: Journal of the Transportation Research Board, no. 1639, pp. 94-101, 1998.

[22] A. K. Schindler, "Prediction of concrete setting," in Proceedings of the RILEM International Symposium on Advances in Concrete through Science and Engineering, eds. J. Weiss, K. Kovler, J. Marchand, and S. Mindess, RILEM Publications SARL, Evanston, Illinois. Citeseer, 2004.

[23] G. Roqueta, L. Jofre, and M. Q. Feng, "Analysis of the electromagnetic signature of reinforced concrete structures for nondestructive evaluation of corrosion damage," IEEE Transactions on Instrumentation and Measurement, vol. 61, no. 4, pp. 1090-1098, 2012.

[24] D. M. Pozar, Microwave engineering. John Wiley \& Sons, 2009.

[25] S. Ramo, J. R. Whinnery, and T. Van Duzer, Fields and waves in communication electronics. John Wiley \& Sons, 2008.

[26] Á. C. Aznar, J. R. Robert, J. M. R. Casals, L. J. Roca, S. B. Boris, and M. F. Bataller, Antenas. Univ. Politèc. de Catalunya, 2004, vol. 3.

[27] G. Roqueta, "Microwave propagative imaging for nondestructive evaluation of concrete structures," Ph.D. dissertation, 2012.

[28] C. A. Balanis, Advanced engineering electromagnetics. John Wiley \& Sons, 1999.

[29] S. Blanch and A. Aguasca, "Seawater dielectric permittivity model from measurements at 1 band," in IGARSS 2004. 2004 IEEE International Geoscience and Remote Sensing Symposium, vol. 2. IEEE, 2004, pp. 1362-1365.

[30] W. B. Weir, "Automatic measurement of complex dielectric constant and permeability at microwave frequencies," Proceedings of the IEEE, vol. 62, no. 1, pp. 33-36, 1974.

[31] T. Kollie, J. Horton, K. Carr, M. Herskovitz, and C. Mossman, "Temperature measurement errors with type $\mathrm{k}$ (chromel vs alumel) thermocouples due to short-ranged ordering in chromel," Review of Scientific Instruments, vol. 46, no. 11, pp. 1447-1461, 1975.

[32] J. R. Baker-Jarvis, M. D. Janezic, J. H. Grosvenor Jr, and R. G. Geyer, "Transmission/reflection and short-circuit line methods for measuring permittivity and permeability- nist," Technical Note (NIST TN)-1355, vol. 1355, no. Technical Note (NIST TN)-1355, 1992.

[33] J. Baker-Jarvis, E. J. Vanzura, and W. A. Kissick, "Improved technique for determining complex permittivity with the transmission/reflection method," IEEE Transactions on microwave theory and techniques, vol. 38, no. 8, pp. 1096-1103, 1990.

[34] A. P. Gregory and R. Clarke, Tables of the complex permittivity of dielectric reference liquids at frequencies up to $5 \mathrm{GHz}$. National Physical Laboratory Teddington, 2001.

[35] H. J. Liebe, G. A. Hufford, and T. Manabe, "A model for the complex permittivity of water at frequencies below 1 thz," International Journal of Infrared and Millimeter Waves, vol. 12, no. 7, pp. 659-675, 1991.

[36] S.-J. Kwon, M. Q. Feng, and S. S. Park, "Characterization of electromagnetic properties for durability performance and saturation in hardened cement mortar," NDT \& E International, vol. 43, no. 2, pp. 86-95, 2010.

[37] "Guidance on radar testing of concrete structures," Concrete Society, 1997.

[38] D. Bucur, N. Militaru, and G. Lojewski, "Models of dielectric materials used in high-speed design by fd 2 td method," in 2013 IEEE 19th International Symposium for Design and Technology in Electronic Packaging (SIITME). IEEE, 2013, pp. 129-133.

[39] I. del Hormigón Estructural, "Ehe-08," Madrid, Ministerio de Fomento, Secretaría General Técnica, 2008.

[40] G. Roqueta, L. Jofre, J. Romeu, and S. Blanch, "Broadband propagative microwave imaging of steel fiber reinforced concrete wall structures," IEEE Transactions on Instrumentation and Measurement, vol. 59, no. 12, pp. 3102-3110, 2010

[41] W. J. McCarter, G. Starrs, and T. M. Chrisp, "Electrical conductivity, diffusion, and permeability of portland cement-based mortars," Cement and Concrete Research, vol. 30, no. 9, pp. 1395-1400, 2000.

[42] P. V. Nikitin and K. Rao, "Performance limitations of passive uhf rfid systems," in 2006 IEEE Antennas and Propagation Society International Symposium. IEEE, 2006, pp. 1011-1014. 


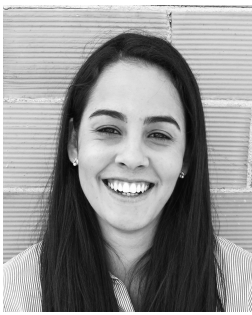

Giselle González-López was born in Havana, Cuba in 1992. She received the B.S. in Telecommunication and Electronics engineering from the Technical University ISPJAE in Havana, in 2015, and the M.S. in Telecommunication engineering from the Technical University of Catalonia (UPC) in Barcelona, in 2018. Currently, she is pursuing a $\mathrm{PhD}$ degree at the Signal Theory and Communications (TSC) Department within the research group of Remote Sensing, Antennas, Microwaves and Superconductivity, Unidad de Excelencia Maria de Maeztu from UPC.

From 2016 to 2017 she worked as an intern at the Research Group of Mobile communications (GRCM) from UPC. Her research interest include but are not limited to embedded systems, media characterization, microwave antenna design, antenna impedance characterization, wireless communications and mobile systems.

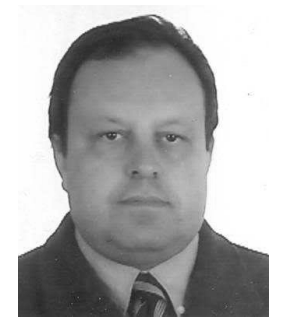

Sebastián Blanch was born in Barcelona, Spain, in 1961. He received the Engineer Degree and $\mathrm{PhD}$ Degree in Telecommunication Engineering from the Polytechnic University of Catalonia (UPC) in Barcelona, Spain, in 1989 and 1996 respectively.

In 1989, he joined the Electromagnetic and Photonics Engineering Group from the Signal Theory and Communications Department (TSC). Currently, he is an Associate Professor at UPC. His research interests are antenna near field measurements, antenna diagnostics, and antenna design.

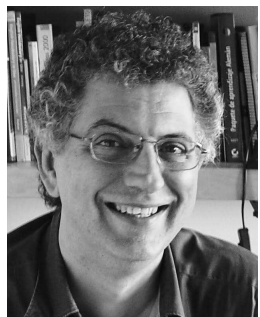

Jordi Romeu (F'12) was born in Barcelona, Spain, in 1962. He received the Engineer Degree and $\mathrm{PhD}$ Degree in Telecommunication Engineering from the Polytechnic University of Catalonia (UPC) in Barcelona, Spain, in 1986 and 1991 respectively. He has been with the Electromagnetic and Photonics Engineering Group from the Signal Theory and Communications Department (TSC) at UPC, since 1985.

Currently, he is a full Professor at UPC, where he is involved in the research of antenna neareld measurements, antenna diagnostics, and antenna design. He joined the Antenna Laboratory, University of California, Los Angeles, in 1999, as a Visiting Scholar. In 2004, he joined the University of California, Irvine, CA, USA. He has authored 50 refereed papers in international journals and 50 conference proceedings and holds several patents. Dr. Romeu received the Grand Winner of the European IT Prize by the European Commission, for his contributions in the development of fractal antennas in 1998.

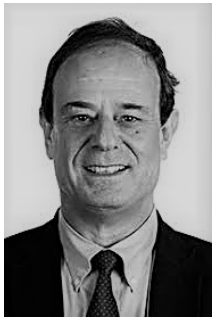

Lluis Jofre (S'79-M'83-SM'07-F'10) was born in Canet de Mar, Spain, in 1956. He received the M.Sc. (Ing.) and Ph.D. (Doctor Eng.) degrees in electrical engineering (telecommunication engineering) from the Technical University of Catalonia (UPC), Barcelona, Spain, in 1978 and 1982, respectively. Since 1982, he has been with the Communications Department, Telecommunication Engineering School, UPC, as an Associate Professor and then as a full Professor since 1989, Director of the UPC-Telefonica Chair on Information Society Future Trends (2003-), Principal Investigator of the 2008-13 Spanish SensingLab Consolider Projet, General Director and Secretary for Catalan Universities and Research (2011-2016), Research Leader of the 2017-2020 CommSens Lab Maria de Maeztu Project and Academic Director of the Consortium for Future Urban Mobility (Carnet). He has authored more than 200 scientific and technical papers, reports, and chapters in specialized volumes. His current research interests include antennas, electromagnetic scattering and imaging, system miniaturization for wireless, and sensing for industrial, scientific and medical applications. 